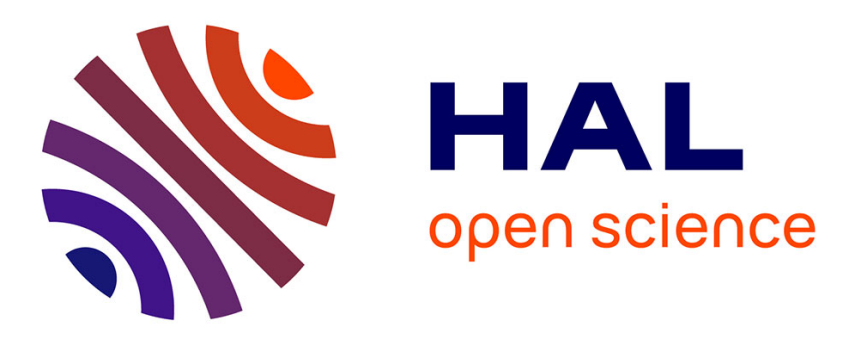

\title{
Imaging of highly inhomogeneous strain field in nanocrystals using x-ray Bragg ptychography: A numerical study
}

Pierre Godard, Marc Allain, Virginie Chamard

\section{To cite this version:}

Pierre Godard, Marc Allain, Virginie Chamard. Imaging of highly inhomogeneous strain field in nanocrystals using x-ray Bragg ptychography: A numerical study. Physical Review B: Condensed Matter and Materials Physics (1998-2015), 2011, 84 (14), pp.144109. 10.1103/PhysRevB.84.144109 . hal-00649563

\section{HAL Id: hal-00649563 https://hal.science/hal-00649563}

Submitted on 26 Sep 2015

HAL is a multi-disciplinary open access archive for the deposit and dissemination of scientific research documents, whether they are published or not. The documents may come from teaching and research institutions in France or abroad, or from public or private research centers.
L'archive ouverte pluridisciplinaire HAL, est destinée au dépôt et à la diffusion de documents scientifiques de niveau recherche, publiés ou non, émanant des établissements d'enseignement et de recherche français ou étrangers, des laboratoires publics ou privés. 


\title{
Imaging of highly inhomogeneous strain field in nanocrystals using x-ray Bragg ptychography: A numerical study
}

\author{
P. Godard, M. Allain, and V. Chamard* \\ Institut Fresnel UMR CNRS 6133, Faculté de Saint-Jérôme case 162, FR-13397 Marseille Cedex 20, France
}

(Received 14 March 2011; revised manuscript received 24 June 2011; published 11 October 2011)

\begin{abstract}
X-ray ptychography is a lensless microscopy method able to provide extended field of view with spatial resolution above the diffraction limit. A series of intensity coherent diffraction patterns measured in the far field is used to obtain the numerical deconvolution between the sample scattering contrast and the illumination function. The measurements are performed with a finite-size beam spot scanned across the sample. The scan step, smaller than the beam size, ensures a high redundancy in the data set, which allows for the convergence of the iterative inversion algorithm. This work explores the possibility to use ptychography for the investigation of strained crystals by means of coherent x-ray Bragg diffraction, taking advantage of the high sensitivity to the atomic displacement fields. The Bragg diffraction scattering contrast is described by an effective complex-valued electron density, where the phase holds the information on the displacement field. The detailed two-dimensional numerical study of Bragg ptychography is presented, both for the known and unknown illumination cases. It demonstrates the high robustness of the ptychographical iterative engine for highly nonhomogeneous strain fields. In particular, the local information is extracted from the individual diffraction patterns to calculate the modulus and phase estimates of the electron density, which are further used to constrain the newly derived algorithm. From this work, it is foreseen that Bragg ptychography when experimentally feasible, will open the way to the nondestructive imaging of strain fields at the nanoscale.
\end{abstract}

DOI: $10.1103 /$ PhysRevB.84.144109

PACS number(s): 68.37.Yz, 42.30.Rx, 61.46.Hk, 61.05.cp

\section{INTRODUCTION}

The ongoing development of nanoscience relies on the new material properties which are obtained when the dimensions of the structures reach the nanometer scale. A detailed description of the structure at the nanoscale is mandatory to understand and monitor these properties: for instance, the functionalities of nanocrystal based devices are highly sensitive to their exact internal chemical composition and strain fields. In this context, hard x-ray diffraction methods are of particular interests: the short wavelength, in the subnanometer range, associated to a large penetration depth allow for a nondestructive characterization of the atomic species and positions. However, the lack of efficient x-ray lens inhibits the possibility for highly resolved x-ray microscopy and leads to constraining modeldependent approaches for the analysis of the x-ray diffraction patterns. ${ }^{1}$

In the past years, lensless $\mathrm{x}$-ray diffraction microscopy has been proposed to overcome this limit. ${ }^{2}$ Instead of lenses, the sample image is obtained from the numerical resolution of the phase problem. ${ }^{3}$ These methods are based on the use of coherent x-ray beams and the measurements of oversampled far-field intensity diffraction patterns. The retrieval of the complex-valued exit wave field at the sample position is obtained with iterative inversion algorithms. ${ }^{4,5}$ Applied in the Bragg geometry, i.e., in the vicinity of a Bragg reflection, the Bragg coherent diffraction imaging (CDI) has allowed for the successful determination of the complete threedimensional (3D) displacement and strain field in a crystal. ${ }^{6}$ However, several limits restrict the general application of this method. As a consequence of the oversampling condition, Bragg CDI is intended for small and isolated crystals, typically in the submicrometer range. This upper limit is mostly due to the actual resolution sustained by the available $\mathrm{x}$-ray two-dimensional (2D) detectors. ${ }^{7,8}$ Furthermore, for weak displacement fields where small phase shifts in the exit field are expected (like, e.g., surface strain), the obtained reconstruction does not allow to discriminate between the illumination wavefront inhomogeneities (as local wavefront curvature) and the crystal displacement field. ${ }^{9}$ The opposite case of highly nonhomogeneous strain fields is also an unsolved problem because the extremely slow convergence of the inversion procedure encountered in that case prohibits the practical use of the method. This latter limit is particularly unfortunate because highly nonhomogeneous strain fields are present in a wide variety of materials like epitaxial nanocrystals (quantum dots, quantum wires), polycrystalline materials with anisotropic elastic constants, embedded nanocrystals for microelectronics applications, etc. Several works have attempted to solve this question. They are all based on the introduction of additional a priori direct space information; therefore they are sample dependant and necessitate an extensive numerical feasibility preanalysis. ${ }^{10-15}$

Recently, a powerful lensless microscopy approach has been demonstrated in the $\mathrm{x}$-ray regime: the ptychography. This method is related to a direct solution of the phase problem first proposed by Hoppe for electron microscopy in the late 60 s. ${ }^{16}$ It is based on the simultaneous inversions of several far-field intensity diffraction patterns obtained for different but overlapping illumination areas onto a large (possibly infinite) sample. ${ }^{17}$ The phase retrieval is numerically ensured with an iterative algorithm like the ptychographical iterative engine $^{18}$ (PIE) or other recently demonstrated approaches. ${ }^{19,20}$ In addition to the possibility of extending the field of view and thereby imaging samples much larger than the transverse coherence lengths of the beam, ${ }^{21-23}$ ptychography also allows for the deconvolution of the sample scattering contrast from the illumination function. ${ }^{20,24,25}$ Quite promising for the Bragg 
case,${ }^{26}$ the experimental demonstration of Bragg ptychography has not been shown yet.

In this paper, we show numerically the potentials of the ptychography approach for imaging highly nonhomogeneous strain fields in a crystal. The detailed problem description and the chosen numerical sample are introduced in Sec. II. Section III describes the algorithmic tools developed for the ptychography approach using the error metric to introduce additional constraints. For Bragg diffraction, the object estimate (modulus and phase) can be extracted and employed as an a priori information. The results obtained for the known illumination case are presented in Sec. IV before the most difficult case of an unknown illumination is described in Sec. V. We finally address the experimental possibilities and the actual limitations in Sec. VI.

\section{COHERENT X-RAY BRAGG DIFFRACTION}

This work investigates the possibility to image strain fields in a crystal using coherent $x$ rays. The basics of coherent $x$-ray Bragg diffraction are summarized in this section before the description of the chosen numerical sample is given.

\section{A. Description of the direct problem}

When only elastic scattering processes are considered, i.e., far from absorption edges, the weak interaction of a monochromatic $\mathrm{x}$-ray beam with a crystal allows for the use of the Born approximation to describe the exit field at the sample position. In the scalar approximation, the exit field $\psi$ at position $\mathbf{r}$ is simply given by the product of the the exact electron density $\rho$ and the incident field (called the probe or illumination function) denoted by $P$ :

$$
\psi(\mathbf{r})=P(\mathbf{r}) \rho(\mathbf{r})
$$

for all positions $\mathbf{r}$ in the sample plane. For measurements performed in the far-field regime, the field at the detector plane is obtained from the Fourier transform of the exit field at the sample plane. The expression for the far-field intensity $I$ evaluated at a position $\mathbf{q}$ of the reciprocal space follows

$$
I(\mathbf{q})=|E(\mathbf{q})|^{2}=|(\mathcal{F} \psi)(\mathbf{q})|^{2},
$$

where $\mathcal{F}$ denotes the Fourier transform operation and $E(\mathbf{q})$ is the field in the Fraunhofer regime. The detector giving only access to the values of $I(\mathbf{q})$ for different $\mathbf{q}$, the phase problem has to be solved to retrieve the exit field. In addition, a detailed knowledge of the illumination function is needed in order to obtain the true electron density $\rho$ from Eq. (1). We note that in CDI experiments, a perfect plane wave is usually assumed; this permits to identify the exit field with the sample scattering contrast.

When the sample is crystalline, the atomic periodicity results in reciprocal space Bragg peaks observed additionally to the forward scattered beam. These Bragg peaks hold information on shape, size, and eventually strain of the crystalline parts. In the case of a strained crystal, the displacement vector field $\mathbf{u}$, which describes the difference between the strained crystal atomic position with regard to the atomic position in an ideally nonstrained crystal, has to be introduced in the electron density distribution. A powerful approximation is so far used

in order to compute the far-field with fast-Fourier-transform routines. ${ }^{28,29}$ It allows expressing the crystal scattering contrast as an effective (complex-valued) electron density:

$$
\rho(\mathbf{r})=|\rho(\mathbf{r})| \exp [i \phi(\mathbf{r})],
$$

with $i=\sqrt{-1}$ and where $|\rho(\mathbf{r})|$ is the density in the unstrained crystal. The phase shift $\phi(\mathbf{r})$, resulting from the displacement field, is given by

$$
\phi(\mathbf{r})=\mathbf{G}_{h k l} \cdot \mathbf{u}(\mathbf{r})
$$

where $\mathbf{G}_{h k l}$, the Bragg vector for the chosen $h k l$ reflection, is related to the considered lattice spacing $d_{h k l}$.

\section{B. The numerical sample: a strained crystal}

The numerical study is performed on the two-dimensional crystalline samples with effective electronic densities shown in Fig. 1. The total matrix dimension is $966 \times 966$ pixels, while the crystal expands over 390 (respectively, 412) pixels along the vertical (respectively, horizontal) direction. The modulus [see Fig. 1(a)] is homogeneous, arbitrarily fixed to unity. In order to investigate the performances of Bragg ptychography in the case of highly nonhomogeneous strain fields, two phase fields are designed, $\phi_{1}$ and $\phi_{2}$ with $\phi_{2}=1.6 \times \phi_{1}$ [see Figs. 1(b) and 1(c)]. In the following, $S_{n}$ refers to the sample whose effective electron density is $\rho(\mathbf{r})=|\rho(\mathbf{r})| \exp \left[i \phi_{n}(\mathbf{r})\right]$.

The parameter of relevance, which allows comparing our work with the available literature concerning diffraction imaging of nonhomogeneous strain field, is the maximal variation of the displacement field gradients observed in the
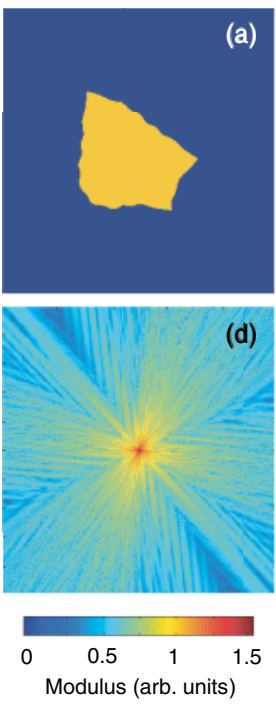

FIG. 1. (Color online) Description of the effective electron density designed for the Bragg ptychography numerical study. The modulus $|\rho(\mathbf{r})|$ is presented in (a) while the phase fields $\phi_{1}$ and $\phi_{2}$ are shown in (b) and (c) for the $S_{1}$ and $S_{2}$ samples, respectively. The corresponding color scales are shown at the bottom: (left) the modulus is in arbitrary units and (middle) the phase is in radians. These scales are common to all electron density maps presented in this work. (d)-(f) Corresponding coherent far-field intensity patterns assuming a plane-wave illumination: (d) strain-free case, (e) [respectively, (f)] $\mathrm{S}_{1}$ (respectively, $\mathbf{S}_{2}$ ) sample, with $\rho(\mathbf{r})=|\rho(\mathbf{r})| \exp \left[i \phi_{n}(\mathbf{r})\right]$ and $n=1$ (respectively, $n=2$ ). The color intensity scale is logarithmic. 
crystal. One can note that the displacement field gradient alone is not sufficient to fully characterize the problem, as a homogenous strain field (with linear displacement field) can exhibit already large (but homogeneous) displacement field gradient. For that crystal, the support-based phase retrieval would be straightforward. When numerical approaches are concerned, the displacement field is given in phase unit, using Eq. (4). The $\phi_{1}$ and $\phi_{2}$ phase fields presented here exhibit a phase gradient variation of 1.2 and $2.1 \mathrm{rad} /$ pixel, respectively. As a matter of comparison, the phase fields of Refs. 10, 12, 13, and 27 for instance, present a phase gradient variation of about $2.1,1.3,1.2$, and $2.1 \mathrm{rad} / \mathrm{pixel}$, respectively. In those works, the authors had to introduce additional constraints to ensure the convergence of the inversion process.

The effects of strain onto the intensity of the coherently diffracted field are illustrated in Figs. 1(e) and 1(f). They present the far-field intensity distributions calculated for the two strained crystals illuminated by a plane wave. These maps have to be compared to the strain-free case shown in Fig. 1(d). The reciprocal space resolution is defined as $2 \pi / \sigma T$, where $T$ is the typical size of the crystal (in pixels) and $\sigma$ is the one-dimensional (1D) oversampling ratio $(\sigma=2.5)$. Strong differences are observed: while Fig. 1(d) presents the expected centrosymmetrical behavior, the diffraction patterns of Figs. 1(e) and 1(f) become asymmetric and hardly show some similarities with the strain-free case. The patterns become elongated and distorted specially in the low and intermediate $\mathbf{q}$ regime. The presence of strong intensity values at the edge of the detector plane points out the necessity to pay a special attention during the inversion process in order to avoid simulation artefacts resulting from unwanted aliasing effects.

\section{THE PTYCHOGRAPHY METHOD}

In the next parts, the PIE will be used to retrieve the effective electron density of the sample from the far-field intensity diffraction patterns (see Sec. IV). Its extended version (ePIE) will be further employed for the simultaneous retrieval of the probe function (see Sec. V). Here, we briefly introduce this algorithm, before we describe the constrained PIE that we use to overcome the encountered limits of the PIE and ePIE. Furthermore, we explain how to make profit from the full set of diffraction patterns to get access to the local information and obtain thereby a reasonable first estimate of the complex electron density.

\section{A. The PIE description}

The PIE allows the retrieval of the scattering contrast of an object that deviates the incident field. It uses a set of diffraction intensity patterns. ${ }^{18}$ A finite-size illumination is used, obtained either by a beam defining aperture located close to the object plane $^{22}$ or by a focusing lens, so that only a small part of the crystal is probed. ${ }^{19}$ The object is scanned across the finite-size beam spot, paying attention to preserve a rather large amount of overlapping area between two successive illuminations. A set of $N$ intensity patterns is obtained, one at each probe position. We denote this data set by $\left\{I_{j}\right\}$ for $j=1, \ldots, N$. An iteration of the PIE is the set of the $N$ updates of the object, with respect to each intensity pattern $I_{j}$. When the probe function is perfectly known, the PIE is initiated with an estimate of the object $\rho_{0,1}$, obtained in most cases from a rough estimate of its support. During the $k$ th iteration, the $j$ th subiteration of the PIE consists in the following four steps: (i) starting with a current object $\rho_{k, j}$, the exit field $\psi_{k, j}$ is calculated for any $\mathbf{r}$ in the sample plane:

$$
\psi_{k, j}(\mathbf{r})=P_{j}(\mathbf{r}) \rho_{k, j}(\mathbf{r})
$$

with $P_{j}$ being the probe position corresponding to the $j$ th measurement. (ii) The far-field distribution $E_{k, j}$ corresponding to the propagation of $\psi_{k, j}$ is obtained from

$$
E_{k, j}(\mathbf{q})=\left(\mathcal{F} \psi_{k, j}\right)(\mathbf{q}) .
$$

(iii) This quantity is corrected so that the calculated intensity matches the experimentally measured data; for any $\mathbf{q}$ in the detector plane, one imposes

$$
\hat{E}_{k, j}(\mathbf{q})=\sqrt{I_{j}(\mathbf{q})} \frac{\left(\mathcal{F} \psi_{k, j}\right)(\mathbf{q})}{\left|\left(\mathcal{F} \psi_{k, j}\right)(\mathbf{q})\right|} .
$$

(iv) The last step allows to update the object estimate, taking into account the probe function and the overlapping condition

$$
\begin{aligned}
\rho_{k, j+1}(\mathbf{r})= & \rho_{k, j}(\mathbf{r})+\beta\left[\frac{\left|P_{j}(\mathbf{r})\right|}{\max _{\mathbf{r}}\left|P_{j}(\mathbf{r})\right|} \frac{P_{j}^{*}(\mathbf{r})}{\left|P_{j}(\mathbf{r})\right|^{2}+\epsilon}\right] \\
& \times\left[\hat{\psi}_{k, j}(\mathbf{r})-\psi_{k, j}(\mathbf{r})\right],
\end{aligned}
$$

where "**" is the conjugation operation, $\hat{\psi}_{k, j}=\mathcal{F}^{-1} \hat{E}_{k, j}$ is the back propagated corrected far field. $\beta$ is the step-length of the update $(\beta>0)$, and $\epsilon$ avoids division by zero.

These four steps are repeated for the $(j+1)$ th probe position. When $j=N$, the $k$ th iteration is over. Typically, a few hundred iterations are needed in order to reach the convergence. The quality of the reconstruction is monitored through the error metric updated at each iteration.

\section{B. Regularization within the PIE: the constrained PIE (cPIE)}

In the following, we describe the constrained PIE (cPIE) that we have to introduce for Bragg ptychography in order to overcome the limits encountered by the PIE. The idea is to enforce the iterative engine to look for a solution close to a relevant estimate. Indeed, the Bragg diffraction data set allows for the possibility to extract an already quite correct preliminary estimate. Interestingly, we note that another method has been proposed to introduce a support constraint to the sample, based on a combination of Fresnel coherent diffraction imaging and ptychography approaches. ${ }^{30}$ In the following, only the modulus regularization is introduced for sake of simplicity. It enforces the solution modulus to resemble an estimate extracted from the diffraction data set (see Sec. IIIC). However, the constrained PIE can be generalized to any other constraints such as the object phase, the object support, the probe continuity, etc.

The vectorial notation is now used as it corresponds to an appropriate discretization of the continuous problem. 
We thus have

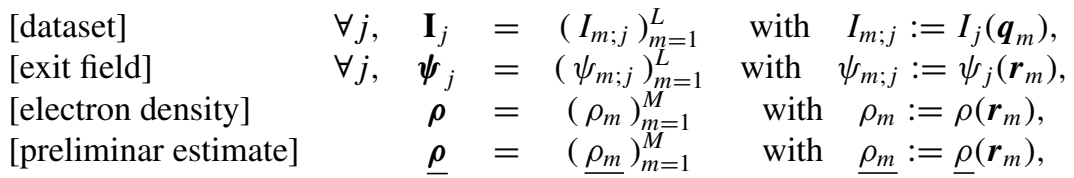

where the subscript $m$ denotes the pixel index and $\rho$ is a good preliminary estimate of the electron density (as obtained for instance in Sec. III C). $L$ and $M$ are the number of pixels of the camera and in the object reconstruction space, respectively. Moreover, we introduce the rectangular matrix ${ }^{31} \boldsymbol{P}_{j} \in \mathbb{C}^{L \times M}$ that leads to the $j$ th exit field induced either by $\rho$ or by $\rho$

$$
\boldsymbol{\psi}_{j}:=\boldsymbol{P}_{j} \boldsymbol{\rho} \quad \text { and } \quad \underline{\boldsymbol{\psi}}_{j}:=\boldsymbol{P}_{j} \underline{\boldsymbol{\rho}} .
$$

The far-field $\mathbf{E}_{j}$ is obtained with the digital Fourier transform (DFT) of the exit field $\psi_{j}$ :

$$
\mathbf{E}_{j}=\boldsymbol{W} \boldsymbol{\psi}_{j},
$$

$$
\begin{aligned}
\forall j, \widehat{\mathbf{E}}_{j} & =\left(\widehat{E}_{m ; j}\right)_{m=1}^{L} \quad \text { with } \\
\widehat{\boldsymbol{\psi}}_{j} & =\left(\widehat{\widehat{\psi}}_{m ; j}\right)_{m=1}^{L} \quad \text { with }
\end{aligned}
$$

The exit field $\widehat{\psi}_{j}$ related to the preliminary estimate constraint and the exit field $\widehat{\psi}_{j}(\rho)$ related to the modulus constraint imposed by the measurements [defined as $\widehat{\boldsymbol{\psi}}_{j}(\boldsymbol{\rho})=\frac{1}{L} \boldsymbol{W}^{\dagger} \widehat{\mathbf{E}}_{j}$ ] can now be used in the cPIE. More precisely, for a given initial guess $\rho^{(0)}$, the cPIE is defined by the following updates for $k=0,1, \ldots$ :

$$
j=1, \ldots N, \quad \begin{aligned}
\boldsymbol{\rho}^{(k, 1)} & :=\boldsymbol{\rho}^{(k)}, \\
\boldsymbol{\rho}^{(k, j+1)} & :=\boldsymbol{\rho}^{(k, j)}-\beta \boldsymbol{D}_{j} \times \boldsymbol{\delta}_{j}\left(\boldsymbol{\rho}^{(k, j)}\right), \\
\boldsymbol{\rho}^{(k+1)} & :=\boldsymbol{\rho}^{(k, N+1)}
\end{aligned}
$$

with $\beta>0$ and

$$
\begin{aligned}
\boldsymbol{\delta}_{j}(\boldsymbol{\rho}) & :=\boldsymbol{P}_{j}^{\dagger}\left[(1+\gamma) \boldsymbol{\psi}_{j}(\boldsymbol{\rho})-\widehat{\boldsymbol{\psi}}_{j}(\boldsymbol{\rho})-\gamma \underline{\boldsymbol{\psi}}_{j}(\boldsymbol{\rho})\right], \\
\boldsymbol{D}_{j} & :=\operatorname{Diag}\left(\frac{\left|P_{m ; j}\right|}{\max _{m}\left|P_{m ; j}\right|} \times \frac{1}{\left|P_{m ; j}\right|^{2}+\epsilon}\right),
\end{aligned}
$$

where $\gamma \geqslant 0$ is a regularization parameter. Note that the iteration (14) reduces to the standard PIE for $\gamma=0$.

It is easy to establish that $\boldsymbol{\delta}_{j}(\boldsymbol{\rho})$ is the gradient (with respect to $\rho^{\dagger}$ ) of the subcriterion $\mathcal{J}_{j}$ defined below. Indeed, following the medical imaging terminology, the iteration (14) where $\boldsymbol{W}$ is the DFT operator. ${ }^{32}$ Finally, the phases associated to the $j$ th exit field and its corresponding far field are defined by

$$
\begin{aligned}
\forall j, m \quad \exp \left[i \varphi_{m ; j}(\boldsymbol{\rho})\right] & :=\frac{E_{m ; j}}{\left|E_{m ; j}\right|}, \\
\exp \left[i \alpha_{m ; j}(\boldsymbol{\rho})\right] & :=\frac{\psi_{m ; j}}{\left|\psi_{m ; j}\right|},
\end{aligned}
$$

where the dependency on the electron density $\rho$ is explicitly indicated.

During the inversion process, these phases are involved in the expressions of the constraints deduced from the intensity diffraction pattern, i.e., the dataset, and from the preliminary estimate, respectively, is an ordered subset algorithm ${ }^{34}$ that aims at minimizing the following penalized criterion:

$$
\mathcal{J}(\boldsymbol{\rho}):=\sum_{j=1}^{N} \mathcal{J}_{j}(\boldsymbol{\rho}) \quad \text { with } \quad \mathcal{J}_{j}(\boldsymbol{\rho}):=\varepsilon_{j}(\boldsymbol{\rho})+\gamma R_{j}(\boldsymbol{\rho}),
$$

where $\varepsilon_{j}(\boldsymbol{\rho})$ is the $j$ th error metric that takes into account the $j$ th measured diffraction pattern

$$
\varepsilon_{j}(\boldsymbol{\rho}):=\sum_{m} \frac{1}{L}\left[\sqrt{I_{m ; j}}-\left|E_{m ; j}(\boldsymbol{\rho})\right|\right]^{2},
$$

and where $R_{j}(\rho)$ is a regularization term that incorporates additional constraints. In the sequel, the following regularization

$$
R_{j}(\rho):=\sum_{m}\left[\left|\psi_{m ; j}(\rho)\right|-\left|\underline{\psi}_{m ; j}\right|\right]^{2}
$$

is adopted in order to constrain the modulus of $\rho$. As for the standard PIE, the global convergence to a local minimizer of the criterion $\mathcal{J}$ is not guaranteed by the iteration (14) (see Appendix A). In addition, it should be underlined that the Euclidean metric $\varepsilon_{j}(\rho)$ is consistent with a thermal (Gaussian) noise assumption in the measurements $\sqrt{I_{m ; j}}$ (see for instance Ref. 35 for a comprehensive presentation of the statistical regularization techniques). Hence, this data-fidelity term [and the iteration (14)] should be modified in order to 
consider a more realistic model like a photon counting process. Nevertheless, this cPIE is considered in the sequel since it is of interest as a natural and simple extension of the standard PIE. We plan to address the global convergence issue and the introduction of a realistic photon noise model in the near future.

\section{First estimate of the strained crystal}

For a better convergence of the PIE, a correct estimate of the exit field is highly desirable as a first guess to initialize the inversion cycle. This relies on a good a priori knowledge of both illumination and object functions. In practice, the probe field may be estimated with a known object. The relevant problem reduces to the initialization of the algorithm with a guessed object $\rho$ as good as possible. We present in this section how to extract this estimate directly from the set of diffraction patterns in order to obtain not only a satisfying estimate for the object modulus but also for the object phase. This approach is similar to the one used in Ref. 19.

\section{The object modulus estimate}

The ptychography approach is based on the measurements of far-field intensities. However, it is still possible to extract the near-field information as obtained in a scanning transmission $\mathrm{X}$-ray microscopy experiment. ${ }^{19}$ This process involves three steps. First, the estimation of the object modulus is performed by monitoring the integrated intensity measured at each beam position onto the sample. The position of each intensity value is identified with the corresponding beam center position. This results in a modulus map sampled on a rough grid determined by the number of illuminations along the two scanning directions. However, the numerical inversion requires to start with an estimate sampled with a pixel size resulting from the inverse of the Fourier space extent. Therefore in a second step, the rough modulus map is interpolated on a finer grid, whose pixel size in the $x$ direction is $2 \pi / \Delta q_{x}$, where $\Delta q_{x}$ is the total shift of the wave-vector transfer sustained by the detection in the $x$ direction-a similar formula holds for the $y$ direction. The last step aims at compensating the broadening resulting from the beam size; as the modulus map is extracted from the intensity pattern ensemble, measured with a non-delta-like probe distribution, the obtained quantity is still convoluted by the probe distribution. The estimate is further truncated in order to keep only the values larger than a threshold (fixed to $85 \%$ in our case). This approach assumes that the electron density of the investigated crystal is homogeneous within the beam spot area, which is true in most cases. ${ }^{10,12,13,27}$

This process is tested on the ptychography data set further used in the inversion process [see Figs. 2(a) and 2(b)]. Each intensity pattern is obtained with an area detector of $256 \times 256$ pixels. The illumination function is a gaussian beam with a $1 / e^{2}$ radius of 73 pixels. Two overlapping ratios $\mathcal{O}$ are considered (see Appendix B for the detailed definition of $\mathcal{O}$ ). For $\mathcal{O}=35 \%$ (respectively, $\mathcal{O}=46 \%$ ), a series of $10 \times 10$ (respectively, $12 \times 12$ ) intensity patterns are obtained with a beam periodically shifted by 78 (respectively, 64) pixels along the two directions. These scan parameters ensure that the whole sample is probed by the different illumination positions.
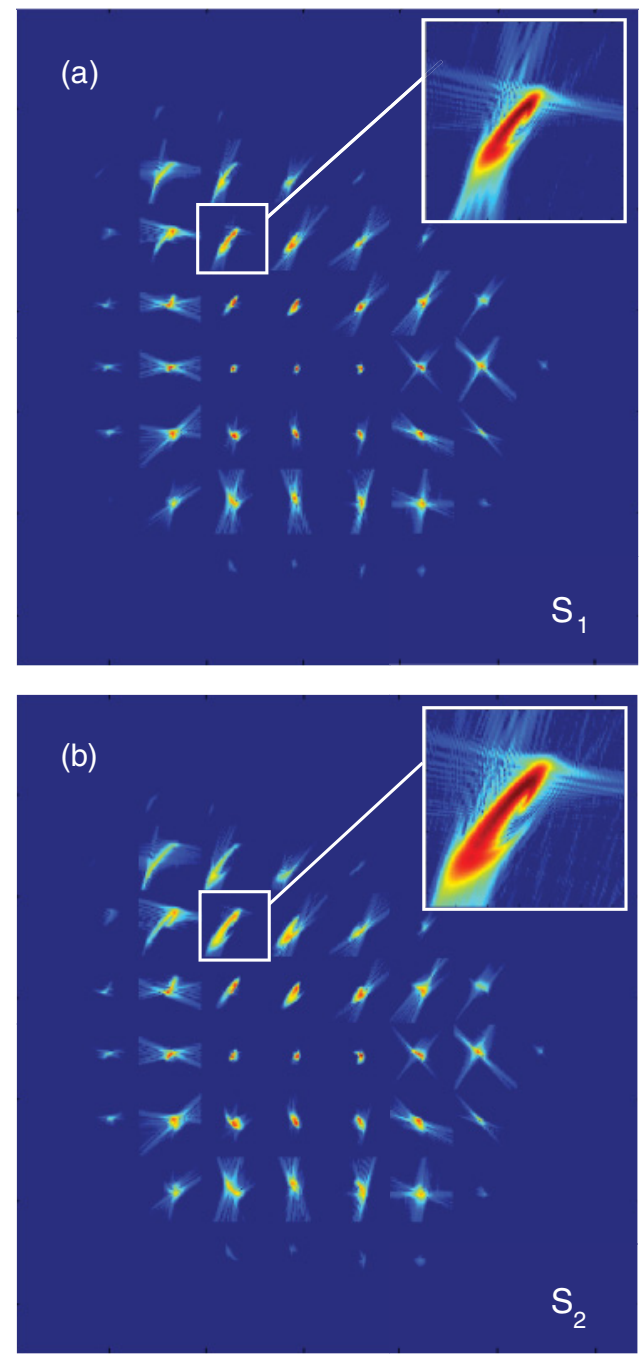

FIG. 2. (Color online) Intensity data set used for the ptychography approach. (a) corresponds to the $S_{1}$ sample and (b) to the $S_{2}$ sample. Each data set is composed of $10 \times 10$ measurements using an area detector of $256 \times 256$ pixels. The illumination is a Gaussian beam (146 pixels in diameter), periodically shifted by 78 pixels along the two directions. The resulting overlapping ratio is $35 \%$.

The modulus estimate process is shown in Figs. 3(a) and 3(a'), where a reasonable agreement with the exact modulus is observed. Further improvements could be obtained with a smaller beam size.

\section{The object phase estimate}

In the case of highly nonhomogeneously strained crystals, the diffraction pattern is mostly governed by the phase associated to the effective electron density, rather than the sample shape itself. Hence, a strong advantage can be taken by extracting the local strain information from the set of diffraction patterns, as suggested in Ref. 19. For each probe position, an approximation of the phase is obtained, based on the relative shift of the Bragg peak with regard to a reference reciprocal space position. Under the Born approximation in the far-field regime, a strain-free crystal produces a Bragg peak, whose position is related to the probed lattice spacing. If the displacement field is linear (homogeneous strain, tilted 

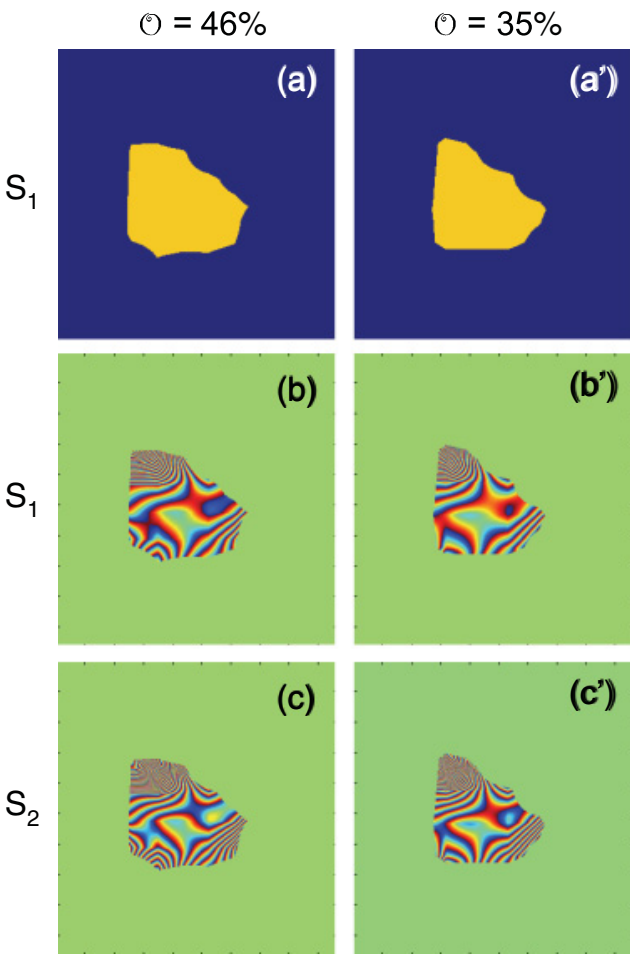

FIG. 3. (Color online) Preliminary estimate of the object $\underline{\rho}$ obtained from the procedure described in Sec. III C. For an overlapping ratio $\mathcal{O}=46 \%$, (a) gives the modulus estimate and (b) [respectively, (c)] shows the phase estimate corresponding to the $S_{1}$ (respectively, $\mathrm{S}_{2}$ ) sample. (a'), (b'), and (c') are the corresponding figures for $\mathcal{O}=35 \%$.

crystal, etc.), the whole Bragg peak is shifted. This shift is linearly related to the displacement field gradient. In order to obtain a phase estimate, one can introduce a simple first-order Taylor expansion of the local displacement field and therefore of the phase of the exit-field at a given $j$ th probe position:

$$
\frac{\psi_{j}(x, y)}{\left|\psi_{j}(x, y)\right|}=\exp \left[i \times\left(\alpha_{0, j}+\alpha_{x, j} x+\alpha_{y, j} y\right)\right]
$$

with $\alpha_{0, j}, \alpha_{x, j}$, and $\alpha_{y, j}$ being real values. The $\alpha_{0, j}$ values depend on the choice of the phase origin: they can not be evaluated in an absolute way because a constant phase shift in the exit field does not change the diffraction pattern. The $\alpha_{x, j}$ and $\alpha_{y, j}$ values are estimated for each diffraction patterns, from the Bragg peak's shift in the $x$ and $y$ directions, respectively: a peak shift of 1 pixel along a given direction is produced by a linear phase with slope of $2 \pi / N$ radians per pixel, $N$ being the number of pixels on the detector along the considered direction. It results in a linear approximation of the exit field phase on a grid determined by the number of illuminations in the $x$ and in the $y$ directions. Then, as for the modulus estimate, these values are interpolated on the finer grid linked to the object reconstruction space; this gives rise to two functions $\alpha_{x}(x, y)$ and $\alpha_{y}(x, y)$ that are the components of the global phase gradient: at the sample position $\left(x_{0}, y_{0}\right)$, one has

$$
\nabla \alpha\left(x_{0}, y_{0}\right)=\left(\begin{array}{c}
\alpha_{x}\left(x_{0}, y_{0}\right) \\
\alpha_{y}\left(x_{0}, y_{0}\right)
\end{array}\right)
$$

the integration is thus straightforward. One fundamental point is that the integration is achieved on the grid of the size of the object; hence, a unique (meaningless) integration constant appears. It allows to preserve a continuous phase behavior, while locally constant gradient values would lead to phase discontinuities located at the boundaries between the domains for $2 \mathrm{D}$ and $3 \mathrm{D}$ systems.

The phase estimate obtained with this method is the phase of the exit field. However, we can identify this phase with the phase of the effective electron density, neglecting thereby the contribution from the eventual wavefront curvature present in the probe. Indeed, the sample is expected to be located at the focus plane of the probe, where the wavefront is planar. In addition, this approach is still valid even if the illumination phase presents some spatial variation; the whole probe being shifted during the ptychographical scan, the curvature is affecting all the diffraction patterns by the same quantity, as long as the modulus of the object is constant. The errors caused by taking a real imperfect illumination are thus acceptable for this phase estimate and will be further corrected by the ptychography iterative algorithm.

The estimate results are presented in Figs. 3(b), 3(c) and 3(b'), 3(c') for the two values of the overlapping ratio and for the two samples. The barycenter of the Bragg peak is used to evaluate the Bragg peak shift; its $\left(Q_{x}, Q_{y}\right)$ coordinates are defined by

$$
\begin{aligned}
Q_{x} & =\frac{\sum q_{x i} I\left(q_{x i}, q_{y i}\right)}{\sum I\left(q_{x i}, q_{y i}\right)}, \\
Q_{y} & =\frac{\sum q_{y i} I\left(q_{x i}, q_{y i}\right)}{\sum I\left(q_{x i}, q_{y i}\right)},
\end{aligned}
$$

where $I\left(q_{x i}, q_{y i}\right)$ is the intensity at the detector pixel with coordinates $\left(q_{x i}, q_{y i}\right)$ and the sum is performed over the detector plane. We observe that the quality of the phase estimate is weakly dependent on the overlapping ratio for the chosen beam size. The phase estimates are rather satisfying, in good agreement with the object phase of Fig. 1. However, some discrepancies are observed, which result from the linear approximation used to model the local phase field. Higher order terms, and in particular cross terms (e.g., $x y$ dependency of the phase field), are at the origin of these discrepancies. In order to solve this problem, we attempted to introduce the second order terms in the Taylor expansion by considering the Bragg peaks broadening. However, no oneto-one relation exists between these second-order coefficients and the variables describing the broadening. Consequently, this approximation was not pursued. Other, slightly more complicated approaches can be found in the literature. ${ }^{36,37}$ However, the simple integration shown here is sufficient in order to provide an estimate of the crystal phase field, which is expected to improve during the inversionprocess.

\section{PTYCHOGRAPHY WITH A KNOWN ILLUMINATION}

The results of the inversion using the PIE method are presented in this part for the two samples $S_{1}$ and $S_{2}$. Noise-free reconstructions with the PIE and cPIE are first considered in an attempt to give an insight into the systematic errors that may occur with these reconstruction methods. Then, more realistic 

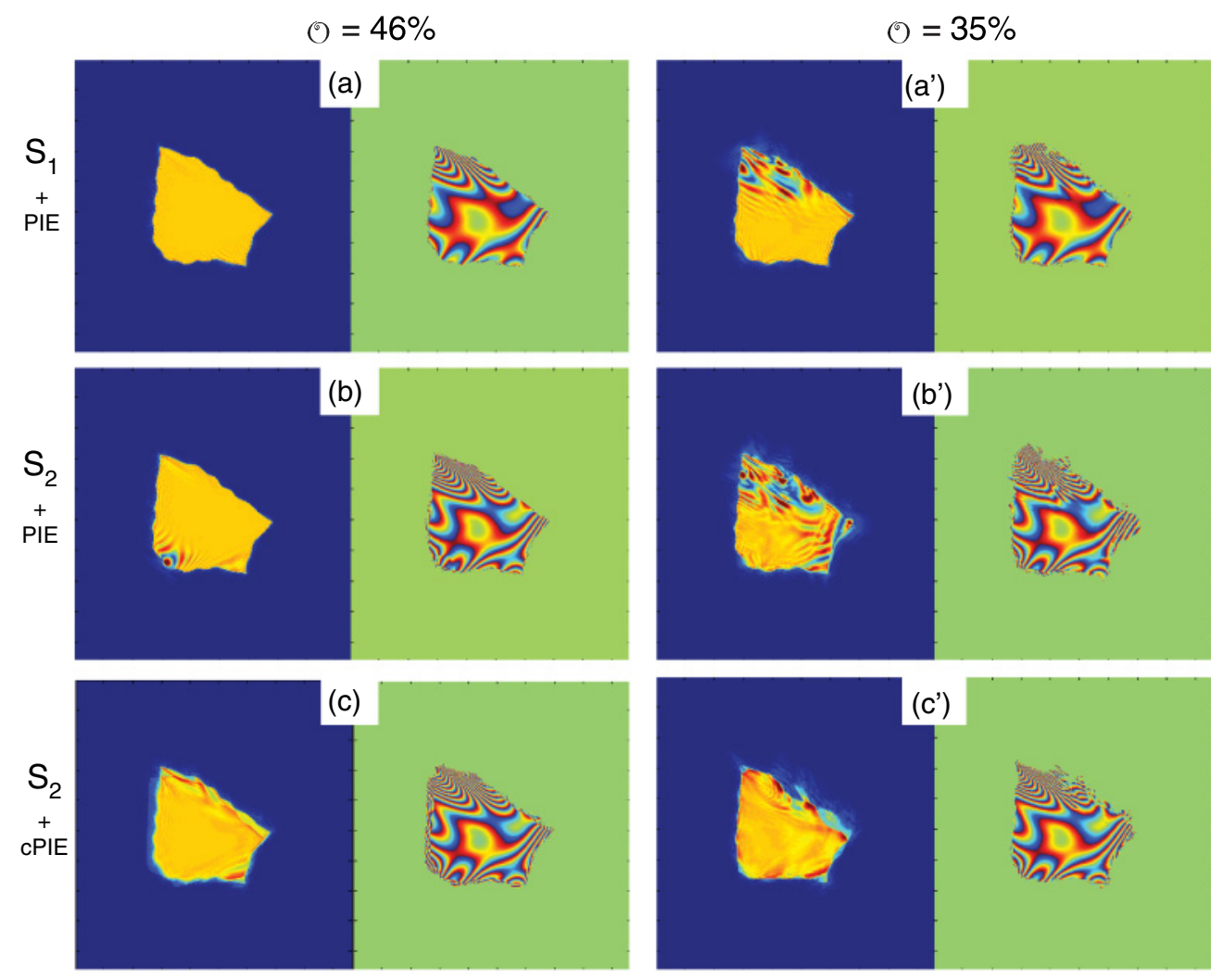

FIG. 4. (Color online) Inversion assuming that the illumination function is known. Solutions retrieved for $\mathcal{O}=46 \%$ and (a) the $\mathrm{S}_{1}$ sample with the PIE, (b) $S_{2}$ sample with the PIE, and (c) $S_{2}$ sample with the modulus cPIE and $\rho$ as initial guess. (a')-(c') Same as (a)-(c) with $\mathcal{O}=35 \%$.

data sets corrupted by photon noise are considered in order to test the performances of the reconstruction algorithms.

In this part, the Gaussian illumination function is supposed to be known. For the inversion, we use $\beta \sim 0.5$ and $\epsilon=10^{-3}$. However, the values of $\beta$ and $\epsilon$ have been found to be not critical for the quality of the inversion results. This argues for a relative stability of the ptychographical method.

\section{A. Phase retrieval results using the PIE}

Figures 4(a), 4(b), 4(a'), and 4(b') show the retrieved modulus and phase for the two samples and the two overlapping values. A total of about 2500 inversion steps are used. The modulus of the initial guess object $\rho^{(0)}$ is obtained from our estimation procedure described in Sec. III C, while its phase is set to zero; we thus have $\rho^{(0)}=|\rho|$. The good agreement between the retrieved quantity and the exact solution in the case of the large overlapping value [see Figs. 4(a) and 4(b)] is a clear demonstration of the robustness and efficiency of the method. This is partly a consequence of the use of a finite-size illumination. This locally results in a less inhomogeneous strain field in comparison to the strain distribution observed in the whole crystal. However, the discrepancies (like e.g., holes in the modulus) observed between the retrieved quantity and the exact solution in the case of the small overlapping value [see Figs. 4(a') and 4(b')] show that the robustness of the method is mostly a consequence of the overlapping condition, which produces additional information by the introduction of various interference terms. ${ }^{17}$ For cases where the inversions are not satisfying, it is interesting to note that the discrepancies occur in the retrieved modulus rather than in the retrieved phase. Strong modulus decreases are observed in the sample regions where the phase gradient variations are the largest. The fact that it is easier to retrieve the phase of the sample rather than its modulus is in accordance with the fact that the far-field data set depends strongly on the object phase (see Fig. 1) but weakly on its modulus, as a consequence of the Fourier transform modeling.

\section{B. Improvements from the use of the phase estimate and the modulus cPIE}

To prevent the formation of holes that appear in the modulus in the previous PIE inversions, the modulus cPIE is now applied with regularization parameters set to $\gamma=0.05$ and 0.2 for $S_{1}$ and $S_{2}$, respectively. Furthermore, the preliminary complex-valued estimate $\rho$ deduced from Sec. III C is used as the initial guess, i.e., $\rho^{(0)}=\rho$. The results are shown in Figs. 4(c) and 4(c'). When the result of the solution reached by the PIE is already almost correct [see Fig. 4(b)], the regularization is not improving the solution, and even introduces a certain amount of bias [see Fig. 4(c)]. On the contrary, a much satisfying solution is found [see Fig. 4(c')] when the PIE is far from converging to the true solution [see Fig. 4(b')]. These behaviors are in agreement with standard results from regularization theory (see for instance Ref. 35 for details). 

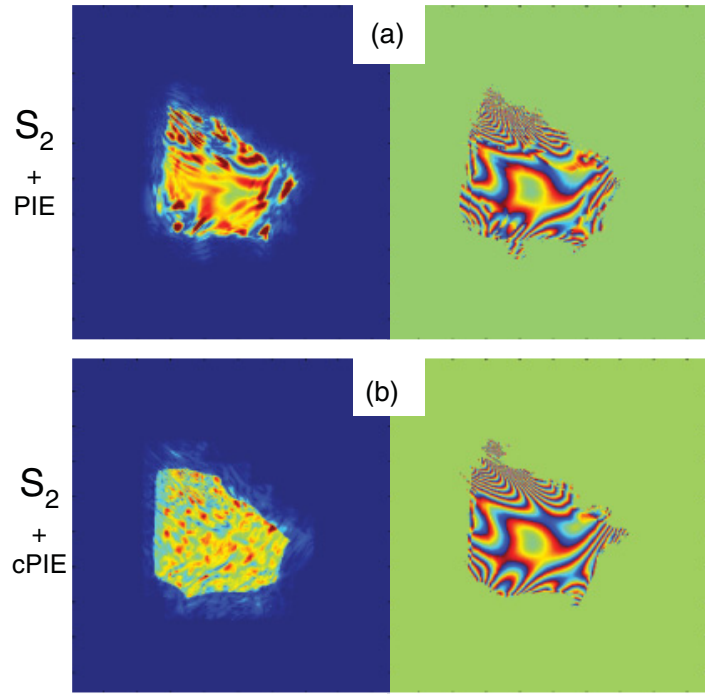

FIG. 5. (Color online) Inversion in the presence of Poisson noise. Solutions retrieved for the $S_{2}$ sample and $\mathcal{O}=46 \%$ by (a) the PIE and (b) the modulus cPIE with $\rho$ as initial guess. The maximum of intensity is set to 21000 photons, corresponding to an average photon value of 200000 per diffraction frame.

\section{Phase retrieval in the presence of noise}

In order to test the robustness of the method in the presence of noise, a Poisson noise is introduced to the intensity data for the $\mathrm{S}_{2}$ sample and $\mathcal{O}=46 \%$. The intensity maximum is 21000 photons, corresponding to an average intensity of about 200000 photons on the whole detection area at each illumination position. These are typical orders of magnitude of photon counts expected for a third generation synchrotron source experiment. The retrieved solutions are shown in Figs. 5(a) and 5(b), using the same methods as the ones used in Figs. 4(b) and 4(c), i.e., PIE and modulus cPIE, respectively. While the PIE fails at retrieving the modulus and phase of the sample scattering contrast, the modulus cPIE (used with the complex-valued preliminary estimate $\rho$ ) leads to a satisfactory reconstruction of the phase. Though, the reconstruction of the modulus is still not perfect. However the proposed cPIE approach leads to an improved solution.

\section{PTYCHOGRAPHY WITH AN UNKNOWN ILLUMINATION}

This part presents the study performed in the case where the illumination function is not perfectly known. One of the characteristics of the ptychography approach is the possibility to retrieve both the sample and the probe functions. ${ }^{20,24,25}$ The deconvolution process is performed in the extended PIE (ePIE) by retrieving successively the object and the probe functions using the same procedure as the one described within the PIE framework.

In the following, the illumination estimate is a truncated Gaussian function whose width has been overestimated by $20 \%$ and whose amplitude has been overestimated by $10 \%$. The optimal inversions are obtained when five iterations on the probe function are followed by five iterations on the object function.

\section{A. Phase retrieval results with the ePIE}

The results of the inversions, performed for the $S_{1}$ and $\mathrm{S}_{2}$ samples and the two overlapping values are presented in Fig. 6. In the most favorable case, the $S_{1}$ sample and the large overlapping ratio [see Fig. 6(a)], the inversion is correct for the retrieved object phase field, while discrepancies are observed in the retrieved modulus, as previously observed in this work. Some of these discrepancies are linked to the chosen scanning step. These are well-known grid artefacts due to the translational periodicity of the illumination function position onto the sample. ${ }^{38}$ These artefacts could in principle be suppressed by the choice of a translational periodic free illumination positioning. For the $S_{1}$ sample, the solution gets noisier with the decrease of the overlapping [see Fig. 6(a')]. The inversion processes performed for the $\mathrm{S}_{2}$ sample are both clearly not satisfying [see Figs. 6(b) and $\left.6\left(b^{\prime}\right)\right]$.

\section{B. Improvements from the use of the phase estimate and the extended modulus cPIE}

In order to improve the inversion in the most critical case observed in the previous section $\left(S_{2}\right.$ sample) the modulus regularization is now introduced. The regularization parameters are $\gamma=0.1$ and $\gamma=0.2$ for $\mathcal{O}=46 \%$ and $\mathcal{O}=35 \%$, respectively. The results, presented in Figs. 6(c) and 6(c'), exhibit clear improvements, although the object modulus is still not fully satisfying. However, the phase field is retrieved with a reasonable agreement.

\section{Phase retrieval in the presence of noise}

As performed in the case of a known illumination, the robustness of the inversion for the unknown illumination case is now tested in the presence of noise. A Poisson noise is introduced to the intensity data, for the $S_{1}$ sample with $\mathcal{O}=46 \%$. The intensity maximum is set to 69000 photons, corresponding to an average intensity of about 440000 photons on the whole detection area at each illumination position. The retrieved solutions are shown in Fig. 7(a) for the ePIE and in Fig. 7(b) when the extended modulus cPIE is used, with $\rho$ as the initial guess. The ePIE clearly fails at retrieving the object. On the contrary, the extended modulus cPIE gives satisfying modulus and phase, although the large regularization parameter $(\gamma=0.5)$ introduces a bias. Additional inversions have been attempted in the most difficult cases, like smaller overlapping ratio, larger phase fields, or smaller numbers of photons, without any success. Better solutions could be sought by using a smaller beam size, a larger overlapping ratio, or a larger detector area.

Nevertheless, these numerical results already demonstrate the new possibilities which can be foreseen from the use of Bragg ptychography in the challenging framework of strain field imaging.

\section{PERSPECTIVES AND LIMITS}

This work numerically demonstrates the possibility to use $\mathrm{x}$-ray Bragg ptychography in order to image nonhomogeneous 

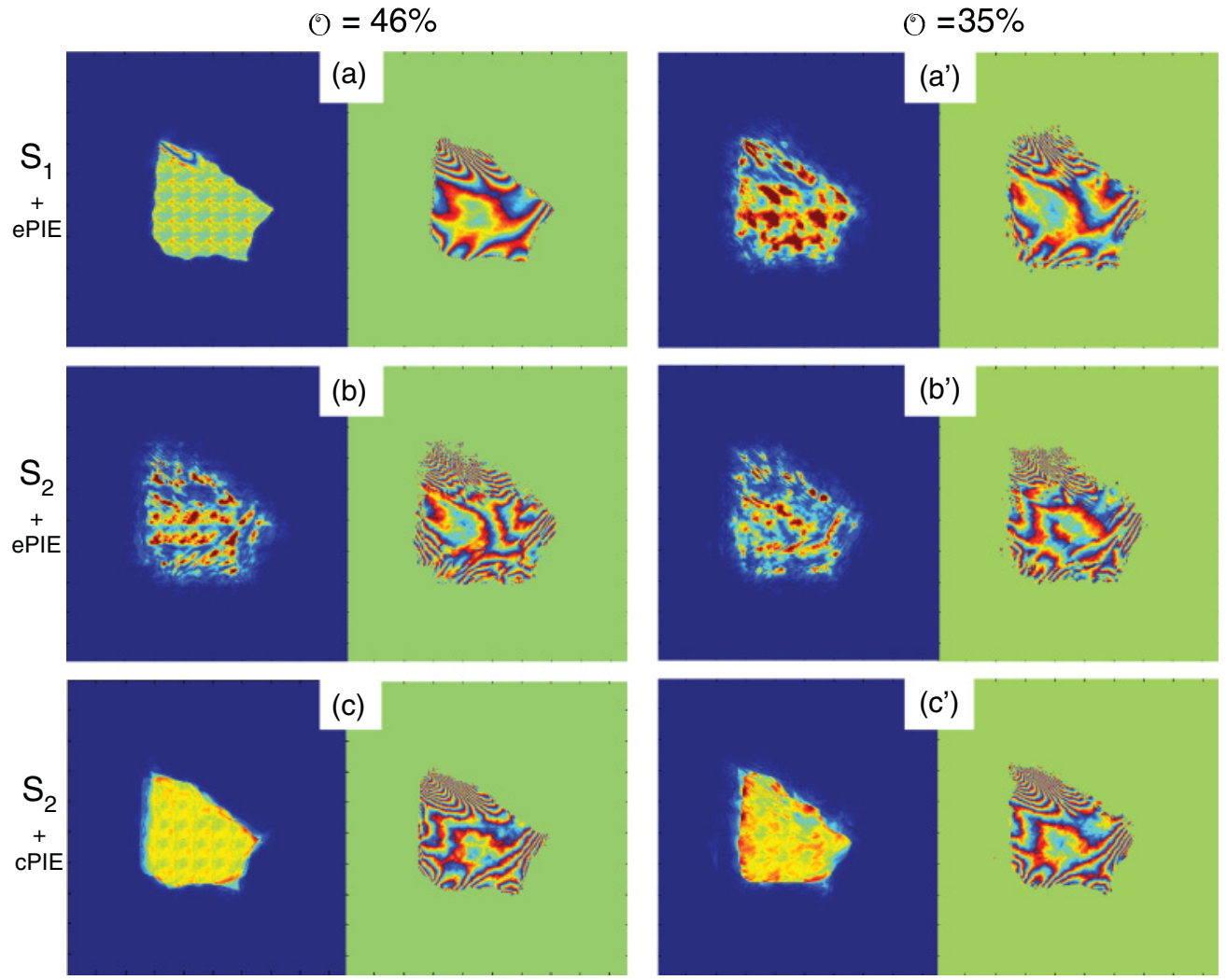

FIG. 6. (Color online) Inversion in the case of an unknown illumination function. Solutions retrieved for $\mathcal{O}=46 \%$ and (a) the $\mathrm{S}_{1}$ sample with the ePIE, (b) $S_{2}$ sample with the ePIE, and (c) $S_{2}$ sample with the extended modulus cPIE and $\rho$ as initial guess. (a')-(c') Same as (a)-(c) with $\mathcal{O}=35 \%$.

strain fields at the nanoscale. The convergence is obtained in a fast way, for known or unknown illumination function cases and is rather robust toward the presence of shot noise.
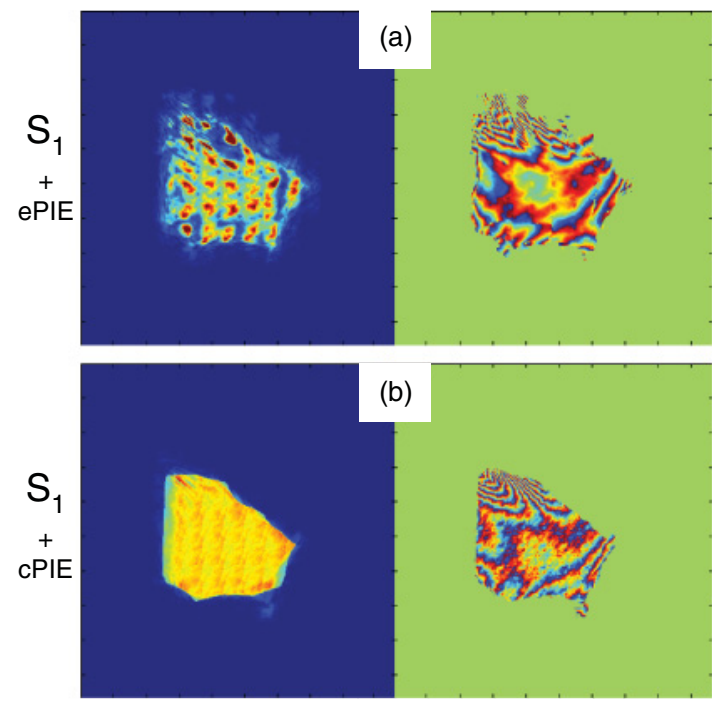

FIG. 7. (Color online) Inversion in the presence of Poisson noise, in the case of an unknown illumination function. Solutions retrieved for the $S_{1}$ sample and $\mathcal{O}=46 \%$ by (a) the ePIE and (b) the extended modulus cPIE with $\rho$ as initial guess. The maximum of intensity is set to 69000 photons, corresponding to an average photon value of 440000 per diffraction frame.
These results suggest that Bragg ptychography will be able to overcome the actual limit of Bragg x-ray coherent diffraction imaging, where the phase retrieval is obtained from a unique and oversampled coherently diffracted intensity pattern. In addition to the strain sensitivity, the Bragg ptychography is allowing for an easy access to the 3D information, generalizing the $2 \mathrm{D}$ approach presented here to the $3 \mathrm{D}$ case without the need for tomographic methods. ${ }^{22}$ Indeed, in the Bragg geometry, a small angular exploration along the rocking curve in the vicinity of the Bragg reflection is transformed into a translation of the wave-vector transfer across the Bragg peak. ${ }^{39,40}$ Therefore using a $2 \mathrm{D}$ detector, the full 3D pattern can be measured for an angular range of about $1^{\circ}$. The resolution is expected to be of the same order of magnitude as the one obtained with CDI methods. However, Bragg ptychography has not been experimentally demonstrated yet, due to some severe technical difficulties. In the following, we discuss the experimental requirements needed to obtain a useful set of Bragg ptychographical data.

The scientific case for the Bragg x-ray ptychography method is governed by the achievements obtained by thirdgeneration synchrotron experimental setups. Beam size, detector resolution, and translation stage specifications have to be considered. The upper limit of the beam size $D_{f}$ has to be compared to the resolution $\delta q$ sustained by the detector: indeed, the coherence condition implies that beam size larger than $D_{f}=2 \pi / \delta q$ can not be used, because it induces a reciprocal space frequency that can not be resolved by the detection resolution. For a detection with a pixel size of about 
$25 \mu \mathrm{m}$, located at $1 \mathrm{~m}$ from the sample, the largest acceptable beam size is about $6 \mu \mathrm{m}$ (at $8 \mathrm{keV}$ ). The lower beam-size limit, in the few $100 \mathrm{~nm}$ range, is not only resulting from the decrease of the total number of coherent photons at the sample position, but also by the achieved accuracy of the translation stage, needed for scanning the sample across the beam. In order to ensure enough redundancy in the set of diffraction patterns, translation steps smaller than about $25 \%$ of the beam size have to be chosen. While piezoelectrical translation stages are able to provide such specifications when they are used horizontally, some difficulties may arise for Bragg diffraction geometry. For a scattering process defined in the vertical plane, the Bragg condition imposes the translation stages to be tilted, resulting in mechanical stresses that affect the translation performances. A more favorable configuration is composed of an already oriented crystal mounted onto the piezo-stage, itself mounted onto a small amplitude cradle, allowing for the measurements of the coherently diffracted intensity distribution along the rocking curve. Other favorable geometries are obtained when the Bragg diffraction is measured in the horizontal plane.

An important conclusion of our numerical study is the need for a reasonable $a$ priori knowledge of the illumination function in order to ensure the convergence of the ePIE for largest strain fields. Preliminary characterization and analysis of the beam properties are desirable. This is of particular importance for experimental setups based on the use of Fresnel zone plate (FZP) focusing optics: the size of the FZP, often larger than the beam transversal coherence length, imposes to reduce the FZP illuminated area in order to ensure a full coherence onto the FZP and therefore at the sample position. Hence, the diffraction-limited illumination condition uses a pair of beam defining slits in front of the focusing optics, necessarily selecting a partial and asymmetric pattern of the FZP. It results in a noteworthy modification of the beam amplitude and phase at the sample position. Fresnel propagation formalism can be used to predict the beam behavior and compare it to experimental observations prior the inversion. ${ }^{41}$ The numerical description of the coherent beam wavefront at the sample position shows that the illumination function can be considered as constant along the $1^{\circ}$ (or less) exploration of the rocking curve.

As a conclusion, this work emphasizes the benefits that will arise from the use of the Bragg ptychography approach; 3D strain mapping at the nanoscale is highly desirable to tackle a large range of material science problems like, e.g., dislocationinduced strain field, nanoscale mechanics in crystalline grain under external stress, internal stress during phase transition, epitaxial growth in nanostructures, biocrystallization, etc. We believe that this method will bring new insights into nanomaterial science in the near future.

\section{APPENDIX A: THE PIE CONVERGENCE}

Following Ref. 34, the iteration (14) can be rewritten

$$
\begin{aligned}
\boldsymbol{\rho}^{(k)} & =\boldsymbol{\rho}^{(k-1)}-\beta \sum_{j=1}^{N} \boldsymbol{D}_{j} \times \boldsymbol{\delta}_{j}\left(\boldsymbol{\rho}^{(k-1, j)}\right) \\
& =\boldsymbol{\rho}^{(0)}-\beta \sum_{l=0}^{k-1} \sum_{j=1}^{N} \boldsymbol{D}_{j} \times \boldsymbol{\delta}_{j}\left(\boldsymbol{\rho}^{(l, j)}\right),
\end{aligned}
$$

where $\delta_{j}(\rho)$ is the gradient of the subcriterion $\mathcal{J}_{j}$ [see Eq. (16)] and with $\beta>0$. Let us assume that this iteration converges toward $\rho^{\star}$; it implies that

$$
\sum_{j=1}^{N} \boldsymbol{D}_{j} \times \boldsymbol{\delta}_{j}\left(\boldsymbol{\rho}^{\star}\right) \equiv \mathbf{0},
$$

otherwise the iterations (A1) would diverge. The above condition is different, in general, from the optimality condition for the full criterion $\mathcal{J}$ that reads

$$
\sum_{j=1}^{N} \boldsymbol{\delta}_{j}\left(\boldsymbol{\rho}^{\star}\right) \equiv \mathbf{0} .
$$

As a result, even if the iterations (14) converge, the resulting estimate is not in general a local minimizer of the criterion (A1). Obviously, the same conclusion holds for the standard PIE iteration since it is just the constrained PIE [see Eqs. (14) and (15)] with $\gamma=0$.

\section{APPENDIX B: THE OVERLAPPING PARAMETER}

The overlapping ratio $\mathcal{O}$ quantifies the redundancy in the ptychography approach. It is a key parameter for the convergence of the PIE. The overlapping ratio depends on the size of the beam and the distance $R$ between two successive illumination positions. In order to take into account the shape of the probe, we consider a surface overlapping ratio instead of a linear one. ${ }^{42}$ The size of the Gaussian beam is identified by its $1 / e^{2}$ radius, which contains $86.5 \%$ of the total intensity. The overlapping ratio compares the area common to two successive illuminations with regard to the number of pixels in the illumination function. Hence, the analytical expression of the overlapping ratio is

$\mathcal{O}=\frac{r_{0}^{2}}{2}\left\{\pi / 2-\arcsin \left(R / 2 r_{0}\right)-\left(R / 2 r_{0}\right)\left[1-\left(R / 2 r_{0}\right)^{2}\right]^{1 / 2}\right\}$,

where $r_{0}$ is the radius of the beam and $R$ is the step distance. For the values chosen in this work, i.e., $r_{0}=73$ pixels and $R=64$ pixels (respectively, $R=78$ ), the overlapping ratio $\mathcal{O}$ is $46 \%$ (respectively, 35\%). As a matter of comparison, within a linear description as the one used in Ref. 42, the linear overlapping ratio value becomes $56 \%$ (respectively, $46 \%$ ).

\footnotetext{
*virginie.chamard@ fresnel.fr

${ }^{1}$ J. Stangl, V. Holý, and G. Bauer, Rev. Mod. Phys. 76, 725 (2004).

${ }^{2}$ J. Miao, P. Charalambous, J. Kirz, and D. Sayre, Nature (London) 400, 342 (1999).
}

${ }^{3}$ D. Sayre, Acta Crystallogr. 5, 843 (1952).

${ }^{4}$ R. W. Gerchberg and W. O. Saxton, Optik 35, 237 (1972).

${ }^{5}$ J. R. Fienup. Appl. Opt. 21, 2758 (1982).

${ }^{6}$ M. C. Newton, S. J. Leake, R. Harder, and I. K. Robinson, Nat. Mater. 9, 279 (2010). 
${ }^{7}$ C. Ponchut, J. Clément, J.-M. Rigal, E. Papillon, J. Vallerga, D. LaMarra, and B. Mikulec, Nucl. Instrum. Methods Phys. Res. Sect. A 576, 109 (2007).

${ }^{8}$ C. Brönnimann, E. F. Eikenberry, B. Henrich, R. Horisberger, G. Huelsen, E. Pohl, B. Schmitt, C. Schulze-Briese, M. Suzuki, T. Tomizaki, H. Toyokawa, and A. Wagner, J. Synchrotron Radiat. 13, 120 (2006).

${ }^{9}$ V. Chamard, M. Dollé, G. Baldinozzi, F. Livet, M. de Boissieu, S. Labat, F. Picca, C. Mocuta, P. Donnadieu, and T. H. Metzger, J. Mod. Opt. 57, 816 (2010).

${ }^{10}$ A. A. Minkevich, M. Gailhanou, J. S. Micha, B. Charlet, V. Chamard, and O. Thomas, Phys. Rev. B 76, 104106 (2007).

${ }^{11}$ A. A. Minkevich, T. Baumbach, M. Gailhanou, and O. Thomas, Phys. Rev. B 78, 174110 (2008).

${ }^{12}$ A. Diaz, V. Chamard, C. Mocuta, R. Magalh aes Paniago, J. Stangl, D. Carbone, T. H. Metzger, and G. Bauer, New J. Phys. 12, 35006 (2010).

${ }^{13}$ N. Vaxelaire, H. Proudhon, S. Labat, C. Kirchlechner, J. Keckes, V. Jacques, S. Ravy, S. Forest, and O. Thomas, New J. Phys. 12, 35018 (2010).

${ }^{14}$ V. Favre-Nicolin, F. Mastropietro, J. Eymery, D. Camacho, Y. M. Niquet, B. M. Borg, M. E. Messing, L. E. Wernersson, R. E. Algra, E. P. A. M. Bakkers, T. H. Metzger, R. Harder, and I. K. Robinson. New J. Phys. 12, 35013 (2010).

${ }^{15}$ M. C. Newton, R. Harder, X. Huang, G. Xiong, and I. K. Robinson, Phys. Rev. B 82, 165436 (2010).

${ }^{16}$ W. Hoppe, Acta Crystallogr. Sect. A 25, 495 (1969).

${ }^{17}$ J. M. Rodenburg and R. H. T. Bates, Philos. Trans. R. Soc. London A 339, 521 (1992).

${ }^{18}$ J. M. Rodenburg and H. M. L. Faulkner, Appl. Phys. Lett. 85, 4795 (2004).

${ }^{19}$ P. Thibault, M. Dierolf, A. Menzel, O. Bunk, C. David, and F. Pfeiffer, Science 321, 379 (2008).

${ }^{20}$ M. Guizar-Sicairos and J. R. Fienup, Opt. Express 16, 7264 (2008).

${ }^{21}$ K. Giewekenemeyer, P. Thibault, S. Kalbfleisch, A. Beerlink, C. M. Kewish, M. Dierolf, F. Pfeiffer, and T. Salditt, Proc. Natl. Acad. Sci. USA 107, 529 (2010).

${ }^{22}$ M. Dierolf, A. Menzel, P. Thibault, P. Schneider, C. M. Kewish, R. Wepf, O. Bunk, and F. Pfeiffer, Nature (London) 467, 436 (2010).

${ }^{23}$ A. Schropp, P. Boye, A. Goldschmidt, S. Hönig, R. Hoppe, J. Patommel, C. Rakete, D. Samberg, S. Stephan, S. Schöder, M. Burghammer, and C. G. Schroer, J. Microsc. 241, 9 (2010).
${ }^{24}$ P. Thibault, M. Dierolf, O. Bunk, A. Menzel, and F. Pfeiffer, Ultramicroscopy 109, 338 (2009).

${ }^{25}$ A. M. Maiden and J. M. Rodenburg, Ultramicroscopy 109, 1256 (2009).

${ }^{26}$ F. Berenguer de la Cuestaa, M. P. E. Wengera, R. J. Beana, L. Bozecb, M. A. Hortona, and I. K. Robinson, Proc. Natl. Acad. Sci. USA 106, 15297 (2009).

${ }^{27}$ A. A. Minkevich, E. Fohtung, T. Slobodskyy, M. Riotte, D. Grigoriev, T. Metzger, A. C. Irvine, V. Novak, V. Holy, and T. Baumbach, Europhys. Lett. 94, 66001 (2011).

${ }^{28}$ S. Takagi, J. Phys. Soc. Jpn. 26, 1239 (1969).

${ }^{29}$ S. Labat, V. Chamard, and O. Thomas, Thin Solid Films 515, 5557 (2007).

${ }^{30}$ D. J. Vine, G. J. Williams, B. Abbey, M. A. Pfeifer, J. N. Clark, M. D. de Jonge, I. McNulty, A. G. Peele, and K. A. Nugent, Phys. Rev. A 80, 063823 (2009).

${ }^{31}$ Indeed, $\boldsymbol{P}_{j}$ retains only the nonzero lines of the diagonal matrix with elements $\left\{P\left(\boldsymbol{r}_{m}-\boldsymbol{r}_{j}\right), m=1, \ldots, M\right\}$.

${ }^{32}$ By definition of the DFT operator see Chap. 1 in Ref. 33, the $j$ th exit field is recovered from its DFT $\boldsymbol{\psi}_{j}$ by the inverse DFT and reads $\boldsymbol{\psi}_{j}=\frac{1}{L} \boldsymbol{W}^{\dagger} \boldsymbol{\psi}_{j}$ with ' $\dagger$ ' the transconjugation operator.

${ }^{33}$ D. F. Elliott, Handbook of Digital Signal Processing (Academic, San Diego, 1987).

${ }^{34}$ S. Ahn, and J. A. Fessler, IEEE T. Med. Imaging 22, 613 (2003).

${ }^{35}$ G. Demoment, IEEE T. Acoust. Speech 37(12), 2024 (1989).

${ }^{36}$ C. Kottler, C. David, F. Pfeiffer, and O. Bunk. Opt. Express 15, 1175 (2007).

${ }^{37}$ M. D. de Jonge, B. Hornberger, C. Holzner, D. Legnini, D. Paterson, I. McNulty, C. Jacobsen, and S. Vogt. Phys. Rev. Lett. 100, 163902 (2008).

${ }^{38}$ M. Dierolf, P. Thibault, A. Menzel, C. M. Kewish, K. Jefimovs, I. Schlichting, K. von König, O. Bunk, and F. Pfeiffer, New J. Phys. 12, 35017 (2010).

${ }^{39}$ G. J. Williams, M. A. Pfeifer, I. A. Vartanyants, and I. K. Robinson, Phys. Rev. Lett. 90, 175501 (2003).

${ }^{40}$ V. Chamard, J. Stangl, G. Carbone, A. Diaz, G. Chen, C. Alfonso, C. Mocuta, and T. H. Metzger, Phys. Rev. Lett. 104, 165501 (2010).

${ }^{41}$ H. Quiney, A. Peele, Z. Cai, D. Paterson, and K. Nugent, Nat. Phys. 2, 101 (2006).

${ }^{42}$ O. Bunk, M. Dierolf, S. Kynde, I. Johnson, O. Marti, and F. Pfeiffer, Ultramicroscopy 108, 481 (2007). 